UM-P-97/26

RCHEP-97/04

\title{
The Mass Spectrum of Light and Heavy Hadrons from Improved Lattice Actions
}

\author{
J.P. Ma and B. H. J. McKellar \\ School of Physics \\ Research Center for High Energy Physics \\ University of Melbourne \\ Parkville, Victoria 3052 \\ Australia
}

\begin{abstract}
:
We use improved lattice actions for glue, light quarks and heavy quarks for which we use lattice NRQCD to compute hadron masses. Our results are in good agreement with experiment, except for charmed hadrons. It seems that charmed quarks are not well approximated as heavy quarks nor as light quarks.
\end{abstract}

PACS numbers: $11.15 \mathrm{Ha}, 12.38 \mathrm{G}, 14.40 \mathrm{G}$ and $14.40 \mathrm{Gx}$ 



\section{Introduction}

A notorious feature of the perturbative theory of lattice QCD is that the perturbation series converges slowly at a moderate lattice spacing. Predictions based only on first two or three terms in the series are in most cases still not reliable. A recent study[1] shows that a partial reason for this is the large contribution from tadpole diagrams, which are absent if one uses dimensional regularization instead of lattice regularization. Based on this the method of tadpole improvement[1] for lattice perturbation series has been suggested. This has many practical implications for studies of lattice QCD. Most importantly it makes the idea of improved actions practicable in simulations of lattice QCD. The idea of improved actions was proposed a long time ago[2], to systematically improve lattice actions using perturbation theory to remove the effect introduced by the finite lattice spacing $a$. If such an improvement really works, one can simulate lattice QCD on a coarse lattice with a lower cost in CPU time, but still obtain reliable results for physics in the continuum limit. Several simulations [3-6], where the tadpole improvement is implemented, already show that this is possible.

With an improved action for glue at the one-loop level and the tree-level improved action for NRQCD the mass spectrum of charmonium is obtained in [3] from lattices with a lattice spacing $a$ between $0.5 \mathrm{fm}$ and $0.2 \mathrm{fm}$, and it agrees with experiment. Similar results were also obtained in other simulations[4], where the mass spectra of bottonium and the $B_{c}$ meson, in addition to that of charmonium, were obtained. With an improved action for light quarks, the D234 action, the mass spectrum of light hadrons is calculated from simulations on coarse lattices[5], and good agreement with experiment is found. In general improved actions are not unique, in [6] another improved action for light fermion, which was proposed long time ago[7], was employed, the mass spectrum of light hadrons was also successfully calculated[6], where the gluonic action improved at tree-level was used. Unlike the widely used SW-action for light quarks[8], in which the effect of $O(a)$ is removed at tree-level, the actions used in these simulations are improved up to $O\left(a^{2}\right)$ at tree-level.

In this work we will present our results for the mass spectrum of light hadrons, strange hadrons, and hadrons containing a b- or c-quark. In calculating the mass spectrum we use the improved action for glue at the one-loop level and the action of [7] for light quarks. For the heavy b- and c-quarks we employ the action of lattice NRQCD. The actions for quarks are all improved at tree-level upto $O\left(a^{2}\right)$. We use the quenched approximation for light quarks. Two lattices are used with the size $6^{3} \times 12$ and $8^{3} \times 16$. Their lattice

spacings determined by the charmonium spectrum are $0.41 \mathrm{fm}$ and $0.23 \mathrm{fm}$ respectively. The whole calculation is performed on two UNIX workstations, which have computational 
power equivalent to that of a high-performance personal computer (like a Pentium Pro 200). Our paper is organized as follows: In Sect.2 we introduce the actions used in this work. Our results for light hadrons and strange hadrons are given in Sect.3. The results of c-flavored and b-flavored hadrons are given in Sect.4. Sect.5 is the summary.

\section{The Improved Actions}

\subsection{The gluonic action:}

We take the one-loop improved action for glue[9], where the action consists of plaquette, rectangle and paralellogram terms and is accurate up to errors of $O\left(\alpha_{s}^{2} a^{2}, a^{4}\right)$. Implementing tadpole improvement the action becomes[3]

$$
\begin{aligned}
S(U) & =\beta \sum_{p l} \frac{1}{3} \operatorname{Re} \operatorname{Tr}\left(1-U_{p l}\right)+\beta_{r t} \sum_{r t} \frac{1}{3} \operatorname{Re} \operatorname{Tr}\left(1-U_{t r}\right)+\beta_{p g} \sum_{p g} \frac{1}{3} \operatorname{Re} \operatorname{Tr}\left(1-U_{p g}\right), \\
\beta_{r t} & =-\frac{\beta}{20 u_{0}^{2}}\left(1+0.4805 \alpha_{s}\right), \quad \beta_{p g}=-\frac{\beta}{u_{0}^{2}} 0.03325 \alpha_{s}, \\
u_{0} & =\left(\frac{1}{3} \operatorname{Re} \operatorname{Tr}\left\langle U_{p l}\right\rangle\right)^{\frac{1}{4}}, \quad \alpha_{s}=-\frac{\ln \left(\frac{1}{3} \operatorname{Re} \operatorname{Tr}\left\langle U_{p l}\right\rangle\right)}{3.06839} .
\end{aligned}
$$

We used this action to generate gluonic configurations at $\beta=7.4$, on a lattice whose size is $8^{3} \times 16$, and also at $\beta=6.8$ with the size $6^{3} \times 12$. The parameter $u_{0}$ is determined by self-consistency. It is 0.8631 and 0.8267 at $\beta=7.4$ and $\beta=6.8$ respectively. The pseudo heat bath method[10] was used to update the links, and the three $S U(2)$ subgroups were updated 3 times in each overall update step. For each lattice we generated 100 configurations for our mass calculations.

\subsection{The improved action for light quarks:}

The improved action proposed in [7] can be written as:

$S_{\text {light }}=-\sum_{x}\left\{m \bar{\psi}(x) \psi(x)+\sum_{\mu} \bar{\psi}(x) \gamma_{\mu} \Delta_{\mu}\left(1-c_{1} \Delta_{\mu}^{(2)}\right) \psi(x)+r \sum_{\mu} \bar{\psi}(x) \Delta_{\mu}^{(2)} \Delta_{\mu}^{(2)} \psi(x)\right\}$,

where $\Delta_{\mu}$ and $\Delta_{\mu}^{(2)}$ are lattice derivatives with the gauge link $U_{\mu}(x)$ :

$$
\begin{aligned}
\Delta_{\mu} \psi(x) & =\frac{1}{2}\left(U_{\mu}(x) \psi(x+\hat{\mu})-U_{\mu}^{\dagger}(x-\hat{\mu}) \psi(x-\hat{\mu})\right), \\
\Delta_{\mu}^{(2)} \psi(x) & =U_{\mu}(x) \psi(x+\hat{\mu})+U_{\mu}^{\dagger}(x-\hat{\mu}) \psi(x-\hat{\mu})-2 \psi(x) .
\end{aligned}
$$

The parameter $c_{1}$ is determined to be $1 / 6$ at tree-level to remove the effect at $O\left(a^{2}\right), m$ is the mass parameter for a quark. The last term in the action with the parameter $r$ 
is introduced in analogy to the Wilson term in the Wilson action to solve the doubling problem of lattice fermions. However, the doubling problem is not totally solved. In the free case one can solve the equation of motion determined by the action to see whether the doublers are removed or not. An analysis in [8], and the analysis in [5] for the D234 action, show that in the low-energy regime the actions describe one particle in the sense that the propagator has only one pole. In high-energy regime there are additional "unphysical" poles. As we are only interested in the low-energy regime as we are in this work, we can expect that the effect from other "unphysical poles" to be negligible. We will take

$r=1 / 6$. With this choice the action is the same as employed in [6], in which it is shown that there is indeed no effect in the low-energy regime which can be related to the "unphysical poles". In this work we used the stabilized biconjugate gradient algorithm[11] to calculate propagators for light quarks. On our lattices this algorithm is at least three times faster than the conventional conjugate gradient algorithm.

\subsection{The Action of Lattice NRQCD}

Heavy quarks whose mass is larger than 1 in lattice units cannot be simulated directly as above with reliable results. To simulate them one uses the heavy quark effective theory, HQET. The formulation of HQET for hadrons with zero velocity is equivalent to that of NRQCD on the lattice, except that the expansion parameters are different. As we will only create hadronic states on lattice with zero space-momenta, we may use lattice NRQCD for heavy quarks like the b- and c-quarks. On the lattice one needs to calculate the propagator of heavy quarks satisfying its appropriate evolution equation. We take the evolution equation proposed in $[12,13]$. The propagator $G(t)$ (where $G(t)=0$ for $t \leq 0$ ) can be calculated on the lattice as:

$$
\begin{aligned}
G(1) & =\left(1-\frac{H_{0}}{2 n}\right)^{n} U_{4}^{\dagger}\left(1-\frac{H_{0}}{2 n}\right)^{n} \delta_{\mathbf{x}, \mathbf{0}}, \\
G(t+1) & =\left(1-\frac{H_{0}}{2 n}\right)^{n} U_{4}^{\dagger}\left(1-\frac{H_{0}}{2 n}\right)^{n}(1-\delta H) G(t),
\end{aligned}
$$

where

$$
\begin{aligned}
H_{0} & =-\frac{\Delta^{(2)}}{2 M_{Q}} \\
\delta H & =-\frac{g}{2 M_{Q}} \sigma \cdot \mathbf{B}+\frac{\Delta^{(4)}}{24 M_{Q}}-\frac{\left(\Delta^{(2)}\right)^{2}}{16 n M_{Q}^{2}}
\end{aligned}
$$

In $(2.5) \Delta^{(4)}$ is the lattice version of the continuum operator $\sum_{i} D_{i}^{4} . M_{Q}$ is the mass parameter for the heavy quark $\mathrm{Q}$. The last two terms in $\delta H$ are the correction terms to remove the effect at order $O\left(a^{2}\right)$. The first term is responsible for spin-splitting in the 
mass spectrum, where $\mathbf{B}$ is the chorommagnetic field. We use definition of $\mathbf{B}$ in terms of gauge links given in [12], and it is also improved up to errors of order $O\left(a^{4}, g^{2} a^{2}\right)$. The parameter $n$ is introduced to avoid numerical instability when high-momentum modes occur. With propagators calculated with Eq.(2.4) we reach an accuracy of order $\frac{1}{M_{Q}}$ in the mass spectrum. With lattice NRQCD we have calculated the mass spectrum of quarkonium at the above $\beta$-values[4], and have determined that at $\beta=7.4$ the mass parameters for $b$ - and $c$-quarks are $M_{b}=4.6$ and $M_{c}=1.4$ respectively. We will use these parameters for our calculations of mas spectra of b- and c-flavored hadrons. We take $n=3$ for c-quark and $n=1$ for b-quark.

In our calculations of quark propagators with the action in Eq.(2.2) or with Eq.(2.4) tadpole improvement is implemented.

\section{The Mass of Light Hadrons}

We create hadronic states on the lattice by using standard local operators $O_{H}(x)$ :

$$
\begin{aligned}
O_{\pi}(x) & =\bar{u}(x) \gamma_{5} d(x), \\
O_{\rho}^{\mu}(x) & =\bar{u}(x) \gamma^{\mu} d(x) \\
O_{P}(x) & =\varepsilon_{a b c} u_{a}(x)\left[u_{b}^{T}(x) C \gamma_{5} d_{c}(x)\right]
\end{aligned}
$$

for $\pi, \rho$ and proton respectively. In Eq.(3.1) $u(x)$ and $d(x)$ stand for $u$ - and $d$-quarks, the indices $a, b$ and $c$ are color indices. With these operators one can measure the corresponding hadron correlations $C_{H}(t)$, in which the hadron has zero space-momentum and its spin is averaged if it has spin. We only use local sources to calculate $C_{H}(t)$, and $u$ - and $d$-quarks are taken to be degenerate in mass. The hadron correlation $C_{H}(t)$ is fitted in a certain time-interval as:

$$
\begin{aligned}
& a_{H}\left(e^{-m_{H} t}+e^{-m_{h}(T-t)}\right) \quad \text { for } H=\pi, \rho, \\
& a_{H} e^{-m_{H} t} \text { with } t<\frac{T}{2}, \quad \text { for } \mathrm{H}=\text { proton, }
\end{aligned}
$$

where $T$ is the lattice size in the time-direction.

Using the lattice configurations on an $8^{3} \times 16$ lattice for $\beta=7.4$ we calculated these hadron correlations varying the mass parameter for light quark from -0.5 to -0.75 , and fit them according to Eq.(3.2). The negative values of the mass parameters simply reflect the difference in sign between the mass parameter and the "Wilson" parameter $r$. The range of light quark mass parameters investigated here are the same order as the mass of the $s$-quark. The fitting window for $C_{\pi}(t)$ and $C_{\rho}(t)$ is chosen from $t=6$ to $t=10$, for 
the proton we can see a plateau in the region of $t=4$ to $t=8$, so we take the window to be from $t=4$ to $t=7$. As examples which show our fit, we plot the measured $C_{H}(t)$ for $\pi$ and $\rho$ with the fitted results in Fig.1A and Fig.1B respectively. Fig. $1 \mathrm{C}$ is the plot for the effective mass of the proton. In all these figures the results are at $m=-0.65$. Our results of fitted masses are given in Table 1.

Table 1

\begin{tabular}{|r|r|r|r|r|r|}
\hline$\beta=7.4$ & $m=-0.5$ & $m=-0.6$ & $m=-0.65$ & $m=-0.7$ & $m=-0.75$ \\
\hline$m_{\pi}$ & $1.142(9)$ & $0.941(11)$ & $0.832(14)$ & $0.713(18)$ & $0.577(27)$ \\
\hline$m_{\rho}$ & $1.362(20)$ & $1.211(24)$ & $1.138(32)$ & $1.068(46)$ & $1.010(95)$ \\
\hline$m_{P}$ & $2.23(9)$ & $1.96(7)$ & $1.82(6)$ & $1.69(7)$ & $1.56(16)$ \\
\hline
\end{tabular}

We also constructed hadron correlations for pion with a minimal lattice momentum $|\mathbf{p}|=\frac{2 \pi}{L}$, where $L$ is the lattice size in the space-direction. Measuring these correlations one can investigate the dispersion relation $E^{2}(\mathbf{p})-m^{2}=c^{2}|\mathbf{p}|^{2}$. We find that $c$ is close to 1. For example, we obtained $c=1.02(7)$ at $m=-0.5$ and $c=1.00(18)$ at $m=-0.75$. This fact indicates that rotational invariance is restored on the lattice. To find the masses in the chiral limit, we fit the hadron masses with:

$$
m_{\rho}=v_{0}+v_{1} m_{\pi}^{2}, \quad m_{P}=u_{0}+u_{1} m_{\pi}^{2}+u_{2} m_{\pi}^{3}
$$

We find that the masses given in Table 1 are well described by these relations as shown in Fig.2. For the mass of the proton we clearly see the effect from the cubic term. With these fits one can extrapolate the masses to the chiral limit.

If we assume that the quark mass is obtained through multiplicative and additive renormalizations of the mass parameter, then the square of $m_{\pi}$ should be linear in the mass parameter $m$. With the data in Table 1 we find good agreement with this relation. Hence we are able to determine the critical mass-parameter $m_{0}$ at which $m_{\pi}=0$. We also determine the mass parameter $m_{s}$ of the strange quark from the experimental value $m_{K}^{2} / m_{\rho}^{2}=0.412$. These parameters at $\beta=7.4$ are:

$$
m_{0}=-0.830(40), \quad m_{s}=-0.6658
$$

With the $m_{s}$ above we calculated the propagator of the s-quark and then the hadron correlations for $K, K^{*}$ and $\phi$, where $C_{K}(t)$ and $C_{K^{*}}(t)$ are calculated with these mass parameters in Table 1 for light quark. The masses obtained for $m_{K}, m_{K^{*}}$ with non-zero mass for the $u$-and $d$-quark are given in Table 2 . 
Table 2

\begin{tabular}{|c|c|c|c|c|c|}
\hline$\beta=7.4$ & $m=-0.5$ & $m=-0.6$ & $m=-0.65$ & $m=-0.7$ & $m=-0.75$ \\
\hline$m_{K}$ & $0.979(12)$ & $0.870(13)$ & $0.814(14)$ & $0.755(16)$ & $0.694(20)$ \\
\hline$m_{K^{*}}$ & $1.239(25)$ & $1.163(29)$ & $1.127(33)$ & $1.092(40)$ & $1.061(50)$ \\
\hline
\end{tabular}

We fit these masses with:

$$
m_{K}^{2}=b_{0}+b_{1}\left(m-m_{0}\right), \quad m_{K^{*}}=c_{0}+c_{1}\left(m-m_{0}\right)
$$

to extrapolate to the limit of zero-mass $u$ - and $d$-quarks. The data and the fitted line are drawn in Fig.3. The data points are well described by the linear fitted line giving confidence in the extrapolation.

Doing the same calculations of $C_{H}(t)$ with our configurations at $\beta=6.8$, for which we choose the region of the light quark mass parameter $m$ from -0.8 to -1.1 , we obtain hadron masses which are given in Table 3.

Table 3.

\begin{tabular}{|c|c|c|c|c|c|}
\hline$\beta=6.8$ & $m=-0.8$ & $m=-0.9$ & $m=-1.0$ & $m=-1.05$ & $m=-1.1$ \\
\hline$m_{\pi}$ & $1.353(9)$ & $1.179(10)$ & $0.986(11)$ & $0.877(13)$ & $0.754(14)$ \\
\hline$m_{\rho}$ & $1.703(21)$ & $1.587(29)$ & $1.470(48)$ & $1.409(62)$ & $1.340(81)$ \\
\hline$m_{N}$ & $2.770(77)$ & $2.523(77)$ & $2.262(79)$ & $2.113(86)$ & $1.941(106)$ \\
\hline$m_{K}$ & $1.218(10)$ & $1.125(10)$ & $1.028(11)$ & $0.977(12)$ & $0.924(12)$ \\
\hline$m_{K^{*}}$ & $1.614(27)$ & $1.554(33)$ & $1.495(43)$ & $1.467(49)$ & $1.439(56)$ \\
\hline
\end{tabular}

At this $\beta$-value we have determined:

$$
m_{0}=-1.233(56), \quad m_{s}=-0.9513 .
$$

With our results we obtain hadron masses in the chiral limit and the mass $m_{\phi}$ which depends only on $m_{s}$. They are given in Table 4 as a ratio to $m_{\rho}$, which is itself given in lattice units.

Table 4

\begin{tabular}{|c|r|r|c|}
\hline & $\beta=6.8$ & $\beta=7.4$ & Exp. \\
\hline$a m_{\rho}$ & $1.198(67)$ & $0.885(45)$ & 1.22 \\
\hline$m_{P} / m_{\rho}$ & $1.16(10)$ & $1.45(11)$ & 0.64 \\
\hline$m_{K} / m_{\rho}$ & $0.63(4)$ & $0.64(3)$ & 1.16 \\
\hline$m_{K^{*}} / m_{\rho}$ & $1.14(7)$ & $1.13(6)$ & 1.32 \\
\hline$m_{\phi} / m_{\rho}$ & $1.27(8)$ & $1.26(9)$ & \\
\hline
\end{tabular}


Having chosen the parameter $m_{s}$ to fit the mass of the $K$, our predictions for the meson masses are in good agreement with experiment, although the quenched approximation was used. The mass of proton obtained at $\beta=7.4$ is $20 \%$ larger than the experimental value, while that from $\beta=6.8$ agrees with the experimental value within the statistical error. A possible reason for the deviation at $\beta=7.4$, apart from the quenched approximation, could be the effect of the finite volume. Another possible reason is the use of a local source in our work, a result of which is that the signal of the ground state in $C_{P}(t)$ is rather weak, and the fitting quality of $C_{P}(t)$ is worse than that of meson propagators. This situation may be improved if smeared sources are used.

From the calculated $\rho$-mass in lattice units we can determine the physical lattice spacing. We obtain:

$$
\begin{aligned}
& a_{\rho}^{-1}=0.64(4) \mathrm{GeV}, \quad \text { at } \beta=6.8, \\
& a_{\rho}^{-1}=0.87(4) \mathrm{GeV}, \quad \text { at } \beta=7.4 .
\end{aligned}
$$

It is instructive to compare the lattice spacings determined from the quarkonium system. In our previous work[4] we obtained:

$$
\begin{array}{lc}
a_{J / \Psi}^{-1}=0.477(5) \mathrm{GeV} & \text { at } \beta=6.8, \\
a_{J / \Psi}^{-1}=0.749(4) \mathrm{GeV}, & a_{\Upsilon}^{-1}=0.861(5) \mathrm{GeV} \quad \text { at } \beta=7.4 .
\end{array}
$$

The lattice spacing at $\beta=7.4$ determined from $\rho$ is close to that obtained from $\Upsilon$, while there is a deviation of $14 \%$ in comparison with $a_{J / \Psi}^{-1}$. At $\beta=6.8$ the deviation is at $30 \%$. Similar lattice spacings were also obtained with D234 action in [5]. Possible reasons for these deviations include: the effects neglected in the quenched approximation being different in different systems, the effect from higher orders in lattice NRQCD can be large for charmonium system because the charm mass is not enough large. In general one should not be surprised that the lattice spacings determined from different systems are different when approximations are used in the calculations.

From the determined dependence of $m_{\rho}$ on $m_{\pi}$ we can obtain the quantity $J=$ $m_{\rho} d m_{\rho}^{2} / d m_{\pi}^{2}$ at the experimental value $m_{\rho} / m_{\pi}=1.8$. This quantity is introduced in [14] to judge the quality of the quenched approximation. For the "real world" it is $J=0.48(2)$, while the "world averaged" result from the quenched approximation is $J=0.37$. From our results we obtain:

$$
J=0.375(87) \text { at } \beta=6.8, \quad J=0.363(63) \text { at } \beta=7.4,
$$

which are in consistent with $J=0.37$. 


\section{The Mass Spectrum of c- and b- flavored Hadrons}

In this section we will present only our results at $\beta=7.4$ in detail. At $\beta=6.8$ there is a problem for simulations of $b$-quark with lattice NRQCD. We find large correlation lengths, so that a significant finite-volume effect should be expected. On this lattice we calculated only masses of c-flavored hadrons and we will mention these results briefly. With the configurations at $\beta=7.4$ we will calculate masses of the hadrons $B^{0}, B^{*}, B_{s}, B_{s}^{*}$ and $\Lambda_{b}$ and masses of the $D^{0}, D^{*}, D_{s}, D_{s}^{*}$ and $\Lambda_{c}$.

For the hadrons we use operators analogous to the operators listed in Eq.(3.1) to create these hadronic states on lattice. The difference is that a light quark field in Eq.(3.1) is replaced by a heavy quark field $Q(x)$ and the field $Q(x)$ only has two nonzero components. The propagation of the field is determined by Eq.(2.4). For light quarks we use the propagators of the last section. For calculating propagators of heavy quarks we use a source smeared by a gaussian:

$$
F(\mathbf{x}, \mathbf{y}, t)=\left[1+\epsilon \sum_{i} \Delta_{i}^{(2)}\right]^{n_{s}}(\mathbf{x}, \mathbf{y}, t)
$$

The smearing parameter $n_{s}$ is fixed at $n_{s}=10$, while the parameter $\epsilon$ is adjusted so that the smearing radius is about the half of the simulated system. Using a smeared source is essential for our simulations. With a local source for heavy quark propagators the signal of hadron correlations is overwhelmed by the statistical noise after 3 or 4 time slices from the source. We only calculate hadron correlations with smeared sources, while the sinks remain unsmeared. The measured hadron propagators are fitted to the form

$$
C_{H}(t) \sim a_{H} e^{-E_{H} t}
$$

where the index $H$ refers to the hadrons mentioned above. With the fitted energy $E_{H}$ one can obtain the hadron mass $M_{H}$

$$
M_{H}=\Delta_{Q}+E_{H},
$$

where $\Delta_{Q}$ is the difference between the renormalized mass of the heavy quark and the zero point energy of the heavy quark on lattice, and it does not depend on the type of hadron. Therefore one can predict the mass difference between two types of hadrons if the $E_{H}$ 's are known.

Although we used a smeared source for heavy quark propagator, the signal is buried in statistical noise after 10 or 11 time slices from the source for mesons and after 8 or 9 time 
slices for baryons. For the meson we already see a plateau in the effective mass. We will take the fit-window for meson propagators from 6 to 9 . For baryons we take the fit-window from 5 to 7 . In Fig.4a, Fig. $4 \mathrm{~b}$ and Fig. $4 \mathrm{c}$ we give our effective mass plots for $B, B^{*}$ and $\Lambda_{b}$ respectively. These plots are for propagators at the mass parameter $m=-0.65$. Our results for $E_{H}$ of various hadrons with nonzero mass of light quarks are given in Table 5 .

Table 5

\begin{tabular}{|c|r|r|r|r|r|}
\hline & $m=-0.5$ & $m=-0.6$ & $m=-0.65$ & $m=-0.7$ & $m=-0.75$ \\
\hline$E_{D^{0}}$ & $1.069(10)$ & $0.990(10)$ & $0.950(9)$ & $0.909(9)$ & $0.863(9)$ \\
\hline$E_{D^{*}}$ & $1.143(8)$ & $1.067(9)$ & $1.030(9)$ & $0.991(10)$ & $0.948(10)$ \\
\hline$E_{\Lambda_{c}}$ & $1.976(16)$ & $1.810(17)$ & $1.727(17)$ & $1.643(15)$ & $1.561(17)$ \\
\hline$E_{B^{0}}$ & $1.043(14)$ & $0.967(12)$ & $0.928(12)$ & $0.894(12)$ & $0.856(13)$ \\
\hline$E_{B^{*}}$ & $1.066(11)$ & $0.999(9)$ & $0.962(9)$ & $0.927(9)$ & $0.891(10)$ \\
\hline$E_{\Lambda_{b}}$ & $1.892(12)$ & $1.713(12)$ & $1.617(14)$ & $1.504(16)$ & $1.381(22)$ \\
\hline
\end{tabular}

For c- and b-flavored hadrons with s-quarks we have:

$$
\begin{aligned}
& E_{B_{s}}=0.920(11), \quad E_{B_{s}^{*}}=0.952(9), \\
& E_{D_{s}}=0.937(9), \quad E_{D_{s}^{*}}=1.018(9) .
\end{aligned}
$$

To extrapolate to the zero light quark mass we assume the dependence of $E_{H}$ on the mass parameter $m$ to be:

$$
E_{H}=E_{H}^{(0)}+E_{H}^{(1)}\left(m-m_{0}\right)
$$

and use this relation to fit our data. In Fig.5A and Fig.5B we show the fits to the data of Table 5. Except for $\Lambda_{b}$, our results in Table 5 can be well fitted with this relation. In Fig.5A the fitted line for $\Lambda_{b}$ is determined only by the first 4 data points. The fit has $\chi^{2}=0.76$. If we include the last point into the fit, the $\chi^{2}$ jumps to 3.39 , and $E_{\Lambda_{b}}^{(0)}$ changes from 1.218(7) to 1.250(9). A reason for this may be the effect of higher orders of $\left(m-m_{0}\right)$ omitted in Eq.(4.5). In our final results we will use the fit with the 4 data point for $\Lambda_{b}$. We obtain the energies at zero mass of light quarks:

$$
\begin{aligned}
& E_{B^{0}}^{(0)}=0.796(4), \quad E_{B^{*}}^{(0)}=0.836(3), \quad E_{\Lambda_{b}}^{(0)}=1.218(7), \\
& E_{D^{0}}^{(0)}=0.800(3), \quad E_{D^{*}}^{(0)}=0.889(3), \quad E_{\Lambda_{c}}^{(0)}=1.428(5) .
\end{aligned}
$$

It is interesting to note that the energies given in Eq.(4.4) and in Eq.(4.6) for b-flavored hadrons are close to those of c-flavored hadrons. These energies $E_{H}$ 's can be expanded in the inverse of $M_{Q}$ with a leading order $M_{Q}^{0}$ and are calculated with an accuracy of $M_{Q}^{-1}$ 
in this work. The above fact indicates that the effect from the next-to-leading order and from higher orders is small in these spin averaged masses. However, as we will see, this is not true for spin-splittings. With the results in Eq.(4.4) and in Eq.(4.6) we are able to predict the mass differences. For doing this we take the lattice spacing determined by the bottonium system for b-flavored hadrons,

$$
a_{b}^{-1}=0.86 \mathrm{GeV} .
$$

This spacing is the same within errors as the lattice spacing determined from $m_{\rho}$ in the last section. We obtain

$$
\begin{aligned}
& M_{B^{*}}-M_{B^{0}}=34(6) \mathrm{MeV}, \quad M_{B_{s}}-M_{B^{0}}=107(13) \mathrm{MeV}, \\
& M_{B_{s}^{*}}-M_{B_{s}}=27(17) \mathrm{MeV}, \quad M_{\Lambda_{b}}-M_{B^{0}}=363(9) \mathrm{MeV} .
\end{aligned}
$$

These results should be compared with the experimental results

$$
\begin{array}{ll}
M_{B^{*}}-M_{B^{0}}=46 \mathrm{MeV}, & M_{B_{s}}-M_{B^{0}}=91 \mathrm{MeV}, \\
M_{B_{s}^{*}}-M_{B_{s}}=47 \mathrm{MeV}, & M_{\Lambda_{b}}-M_{B^{0}}=363 \mathrm{MeV} .
\end{array}
$$

We find that our results for $M_{\Lambda_{b}}-M_{B^{0}}$ and $M_{B_{s}}-M_{B^{0}}$ agree well with experimental results, while the spin-splitting $M_{B^{*}}-M_{B^{0}}$ and $M_{B_{s}^{*}}-M_{B_{s}}$ are not in such good agreement, but differ by less than 2 standard deviations from the observed values. The value of $M_{B^{*}}-M_{B^{0}}$ is $28 \%$ lower than experimental value, but it agrees with the result from a large and fine lattice[15].

For c-flavored hadrons we take the lattice spacing determined by the charmonium system:

$$
a_{c}^{-1}=0.75 \mathrm{GeV}
$$

and obtain

$$
\begin{aligned}
& M_{D^{*}}-M_{D^{0}}=67(5) \mathrm{MeV}, \quad M_{D_{s}}-M_{D^{0}}=103(9) \mathrm{MeV}, \\
& M_{D_{s}^{*}}-M_{D_{s}}=66(14) \mathrm{MeV}, \quad M_{\Lambda_{c}}-M_{D^{0}}=471(6) \mathrm{MeV} .
\end{aligned}
$$

The experimental results are:

$$
\begin{array}{ll}
M_{D^{*}}-M_{D^{0}}=143 \mathrm{MeV}, & M_{D_{s}}-M_{D^{0}}=104 \mathrm{MeV} \\
M_{D_{s}^{*}}-M_{D_{s}}=144 \mathrm{MeV}, & M_{\Lambda_{c}}-M_{D^{0}}=420 \mathrm{MeV} .
\end{array}
$$

Our value for $M_{D_{s}}-M_{D^{0}}$ agrees well with the experimental value, but the predicted $M_{\Lambda_{c}}-M_{D^{0}}$ is $12 \%$ larger the experimental value. More worse are the spin-splittings which are much lower than experimental values. Such discrepancies may be expected as we used lattice NRQCD upto order of $1 / M_{Q}$. For $c$-quarks the effect from higher orders is 
very significant because the charm mass is not very large. A recent study of quarkonium systems[16] also shows that the effect of higher order terms in $1 / M_{Q}$ is large. We also have calculated masses of c-flavored hadrons at $\beta=6.8$ with similar results. It shows that the action for lattice NRQCD employed here is not accurate enough for calculations of the spin-splitting for c-flavored hadrons.

To obtain the absolute mass of a b- or c-flavored hadron we need to know $\Delta_{Q}$ in Eq.(4.3). This quantity can be calculated perturbatively. It can also be extracted by studying the mass spectrum of the quarkonium system. We extracted this number from our previous study at $\beta=7.4$ :

$$
\Delta_{c}=1.59(11), \quad \Delta_{b}=5.25(18) .
$$

It should be noted that effects from the spin-dependent interaction are neglected in the extraction. Adding the spin-dependent interaction has little effect on these numbers, and also these numbers have a quite large uncertainty. Using Eq.(4.13) we obtain

$$
M_{D^{0}}=1.79(8) \mathrm{GeV}, \quad M_{B^{0}}=5.20(15) \mathrm{GeV}
$$

where the lattice spacings in Eq.(4.10) and in Eq.(4.7) are used respectively. These predictions are in good agreement with the experimental values:

$$
M_{D^{0}}=1.864 \mathrm{GeV}, \quad M_{B^{0}}=5.278 \mathrm{GeV} .
$$

However, the accuracy of our predictions is not good because of the large statistical error of $\Delta_{Q}$.

\section{Summary}

In this work we used improved actions for glue and quarks to calculate masses of light, strange, c- and b- flavored hadrons, where for c- and b-quarks we employed lattice NRQCD. The actions for quarks are improved at tree-level to remove the effect at order of $O\left(a^{2}\right)$, while the gluonic action is improved at one-loop level. Tadpole improvement is implemented. The results obtained in this work for light and strange mesons are in agreement with experimental results. We obtain the mass spectrum of b-flavored hadrons with improved actions on a coarse lattice with the lattice spacing as $0.23 \mathrm{fm}$, and it is in agreement with experiment. For c-flavored hadrons the large effect from higher orders neglected in lattice NRQCD prevents us from obtaining spin-splitting in mass comparable 
with experiment. In this work we have shown that improved actions with tadpole improvement can be used not only in light hadron sectors and in quarkonium systems as shown already in previous studies, but also works in the case of hadrons containing one heavy quark.

\section{Acknowledgment:}

This work is supported by Australia Research Council. We thank our computer manager Dr. M. Munro for help with the implementation of our calculations. 


\section{Reference}

[1] G.P. Lepage and P.B. Mackenzie, Phys. Rev. D48 (1993) 2250

[2] K. Symanzik in Mathematical Problems in Theoretical Phyics, ed. R. Schrader et al. Lecture Notes in Physics 153 (Springer Berlin 1982)

Nucl. Phys. B226 (1983) 187

[3] M. Alford et. al. Phys. Lett. B361 (1995) 87

[4] J.P. Ma and B.H.J. McKellar, Melbourne Preprint UM-P-96/55

[5] M. Alford, T. Klassen and G.P. Lepage, Nucl. Phys.(Proc. Suppl.) B47 (1996) 370

[6] H.R. Fiebig and R.M. Woloshyn, Phys. Lett. B385 (1996) 273

[7] H. Hamer and C.M. Wu, Phys. Lett. B133 (1983) 351

W. Wetzel, Phys. Lett. B136 (1984) 407

T. Eguchi and N. Kawamoto, Nucl. Phys. B237 (1984) 609

[8] B. Sheikholeslami and R. Wohlert, Nucl. Phys. B259 (1985) 572

[9] M. Lüscher and P. Weisz, Comm. Math. Phys. 97 (1985) 59

[10] N. Cabibbo and E. Marinari, Phys. Lett. B119 (1982) 387

[11] A. Frommer et.al. Int. J. Mod. Phys. C5 (1994) 1073

[12] G.P. Lepage et al, Phys. Rev. D46 (1992) 4052

[13] C.T.H. Davies et al, Phys. Rev. D50 (1994) 6963

[14] P. Lacock and C. Michael, Phys. Rev. D52 (1995) 5213

[15] A. Ali Khan et al., Phys. Rev. D53 (1996) 6433

[16] H.D. Trottier, Preprint SFU HEP-131-96, hep-lat 9611026 


\section{Figure Caption}

Fig.1A: The propagator $C_{\pi}(t)$ with $m=-.65$. The points with error are measured, the line is from the fit. The $\mathrm{x}$-axis is for $t$.

Fig.1B: The propagator $c_{\rho}(t)$ with $m=-.65$.

Fig.1C: The effective mass plot for the proton propagator with $m=-.65$, the effective mass is defined as $m_{\mathrm{eff}}=\ln \left(C_{p}(t-1) / C(t)\right)$. The x-axis is for $t$.

Fig.2: The chiral extrapolation for $\rho$ and proton. The upper line with data points is for proton. The lower line with data points is for $\rho$. The x-axis is read to be $m_{\pi}^{2}$.

Fig.3: The chiral extrapolation for strange meson.

Fig.4A: The effective mass plot for $B$ with $m=-0.65$.

Fig.4B: The effective mass plot for $B^{*}$ with $m=-0.65$.

Fig.4C: The effective mass plot for $\Lambda_{b}$ with $m=-0.65$.

Fig.5A: The chiral extrapolation for b-flavored hadrons. The upper line with data points is for $\Lambda_{b}$, the middle one with data points is for $B^{*}$, the lower one with data points is for $B$. The $\mathrm{x}$-axis is read as $m$.

Fig.5B: The chiral extrapolation for c-flavored hadrons. The upper line with data points is for $\Lambda_{c}$, the middle one with data points is for $D^{*}$, the lower one with data points is for $D$. The $\mathrm{x}$-axis is read as $m$. 


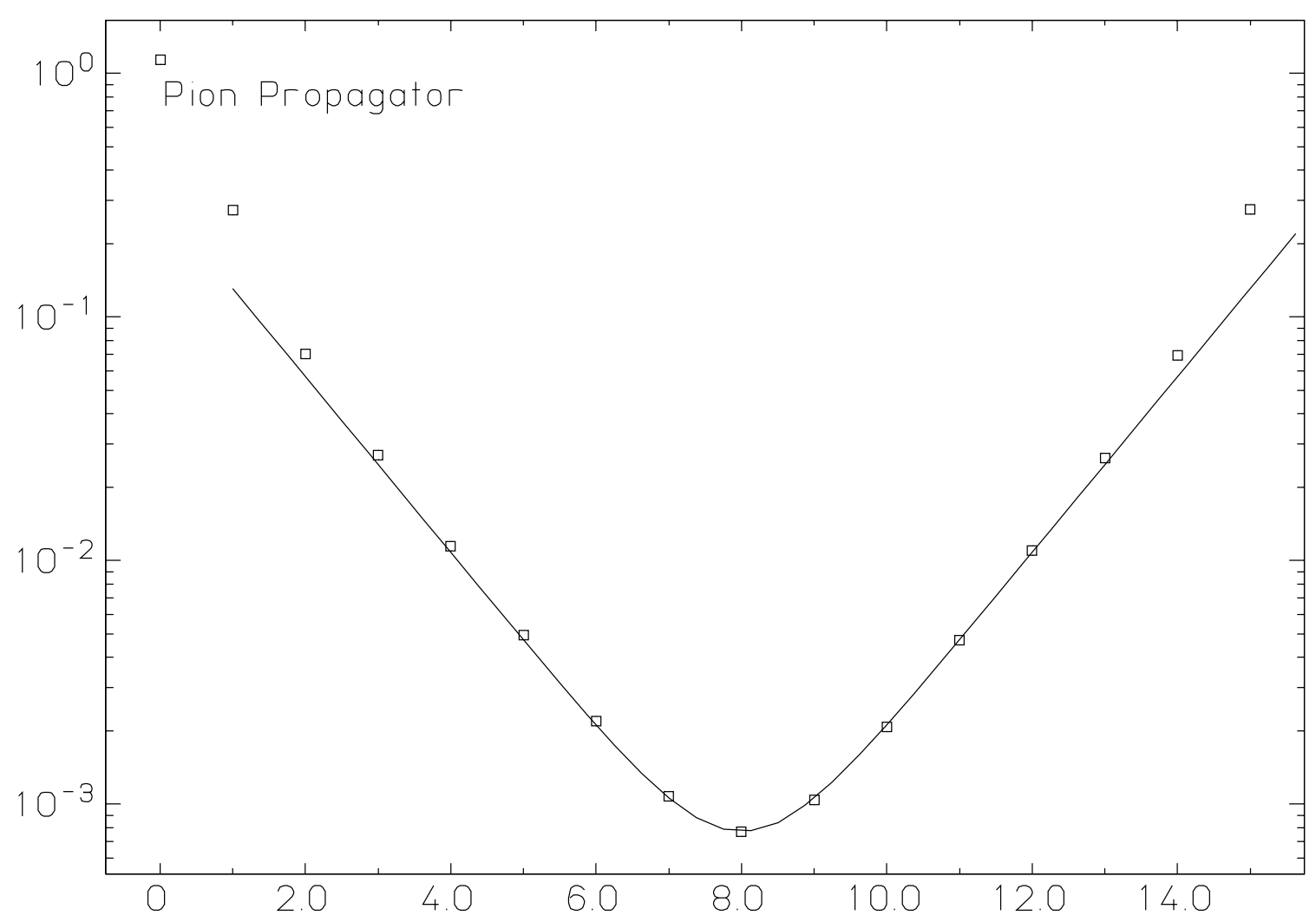

Fig. $1 \mathrm{~A}$

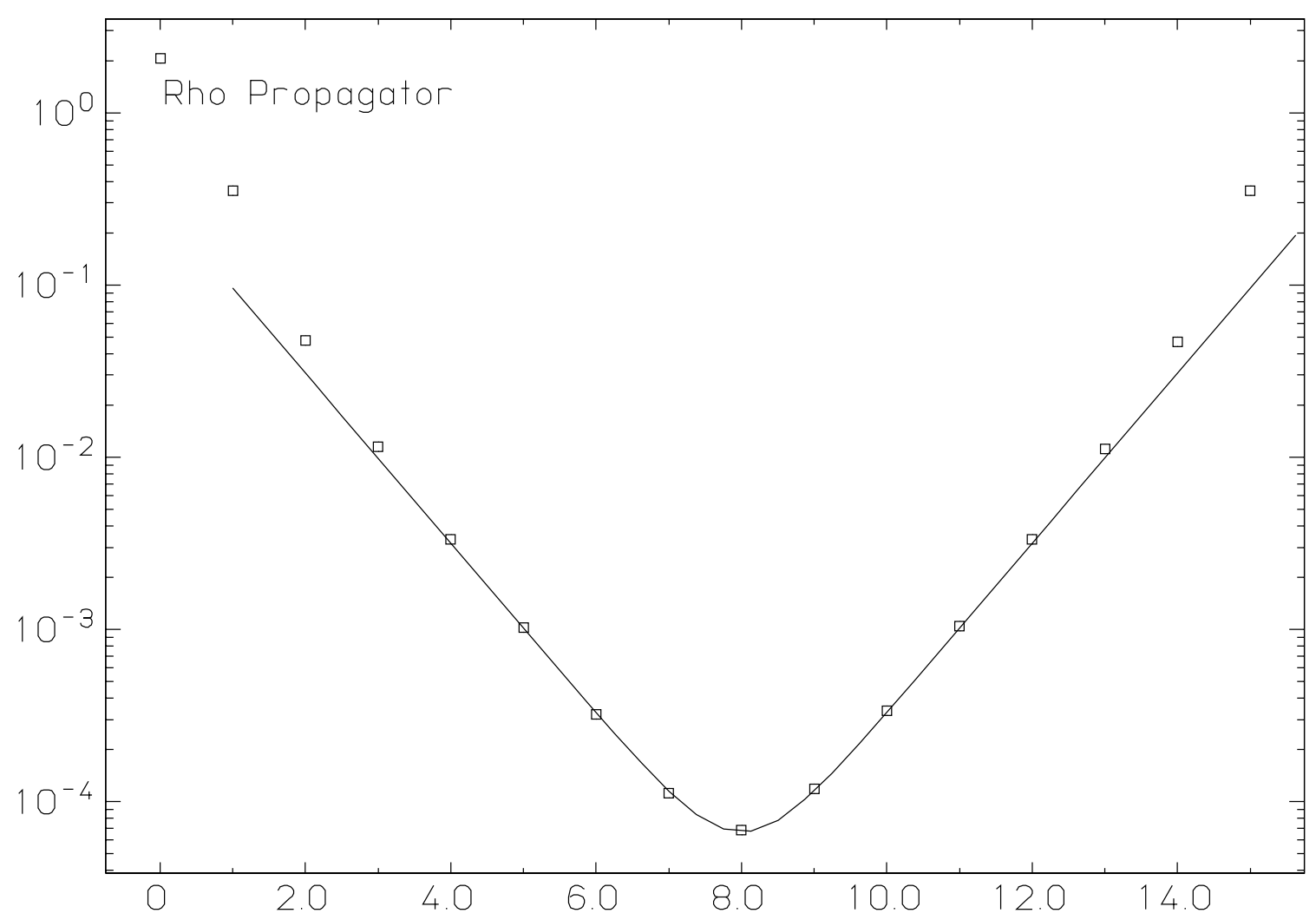

Fig. 1 B 


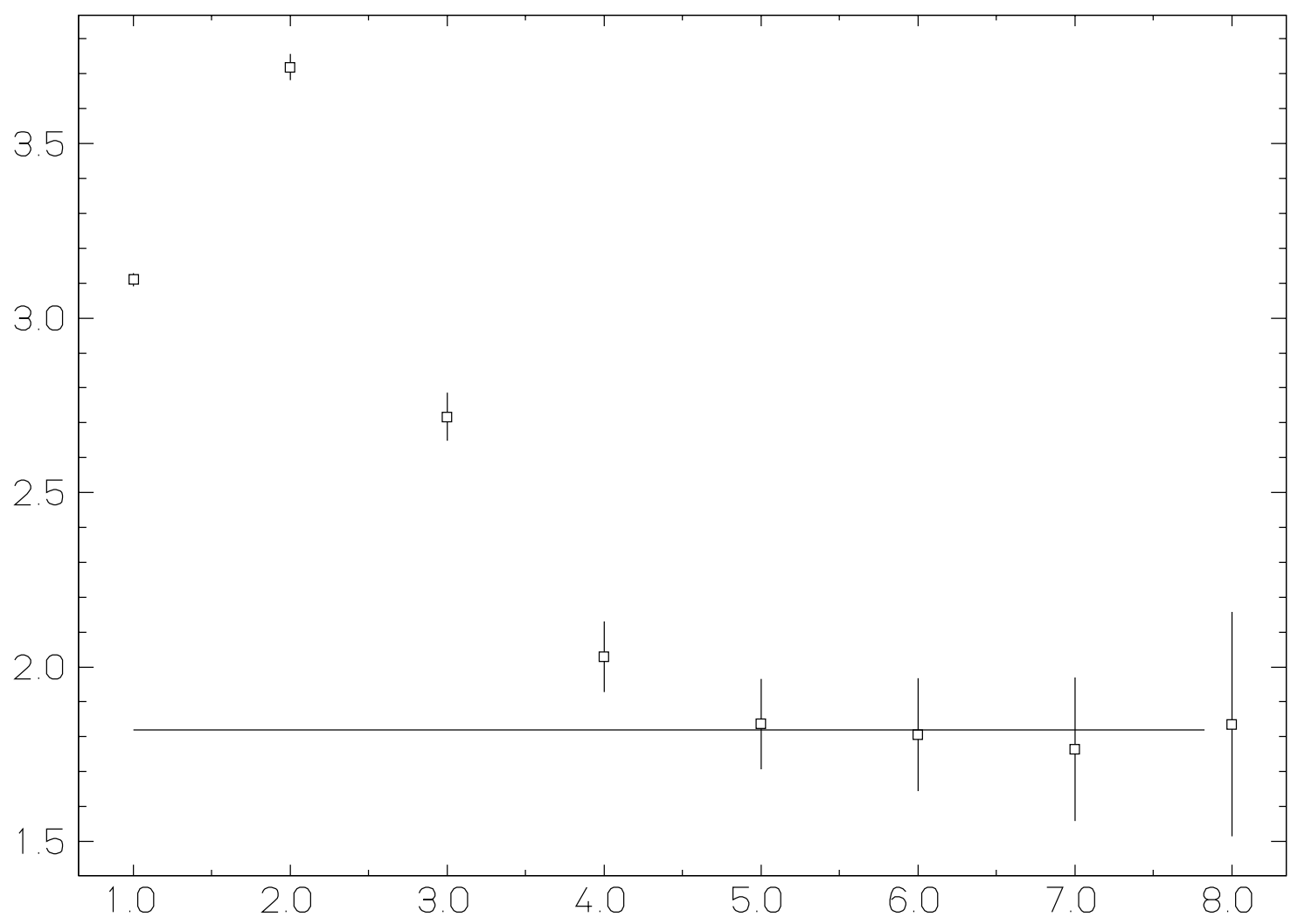

Fig. $1 \mathrm{C}$ 


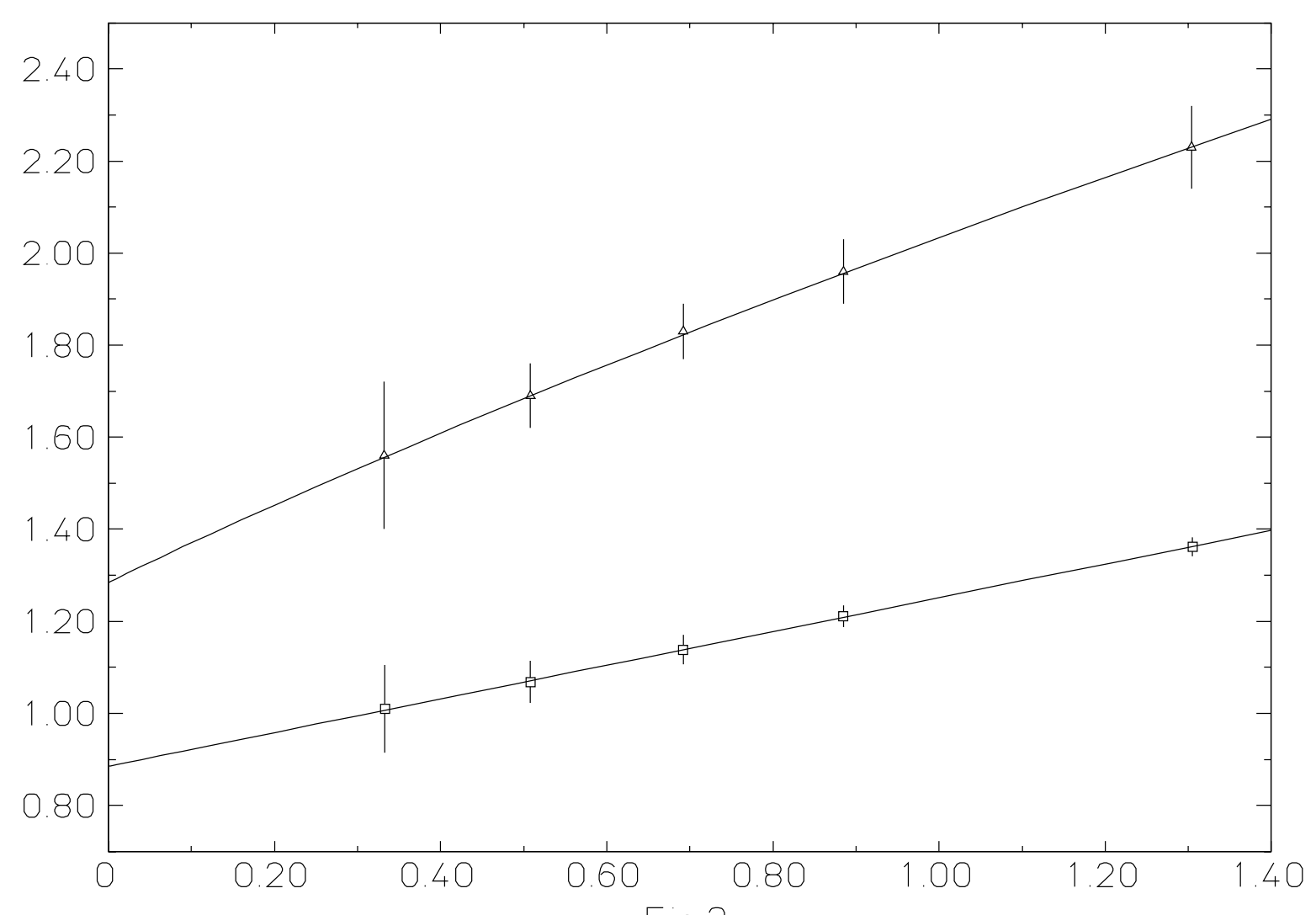

Fig.2

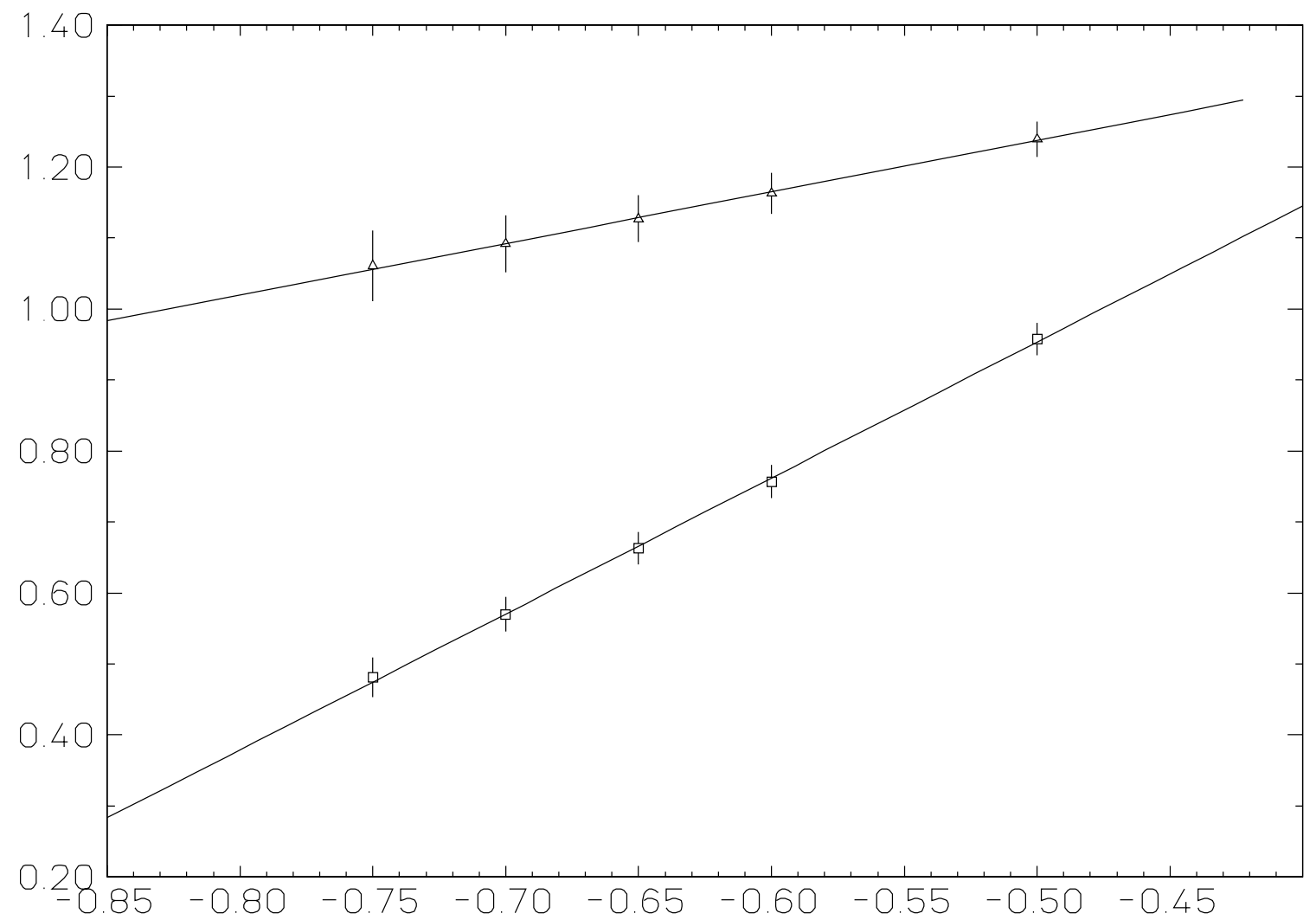

Fig. 3 


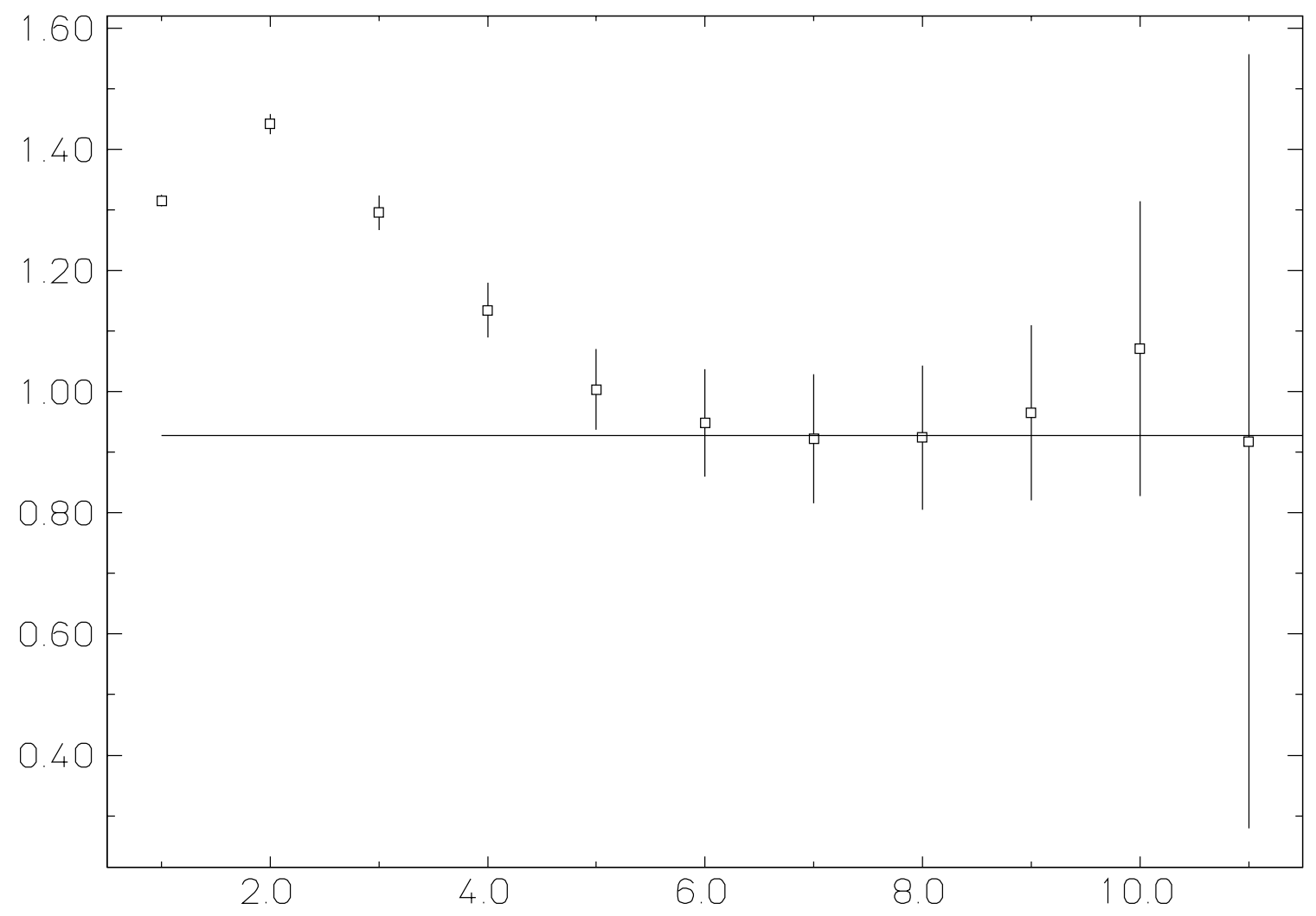

Fig. 4 A

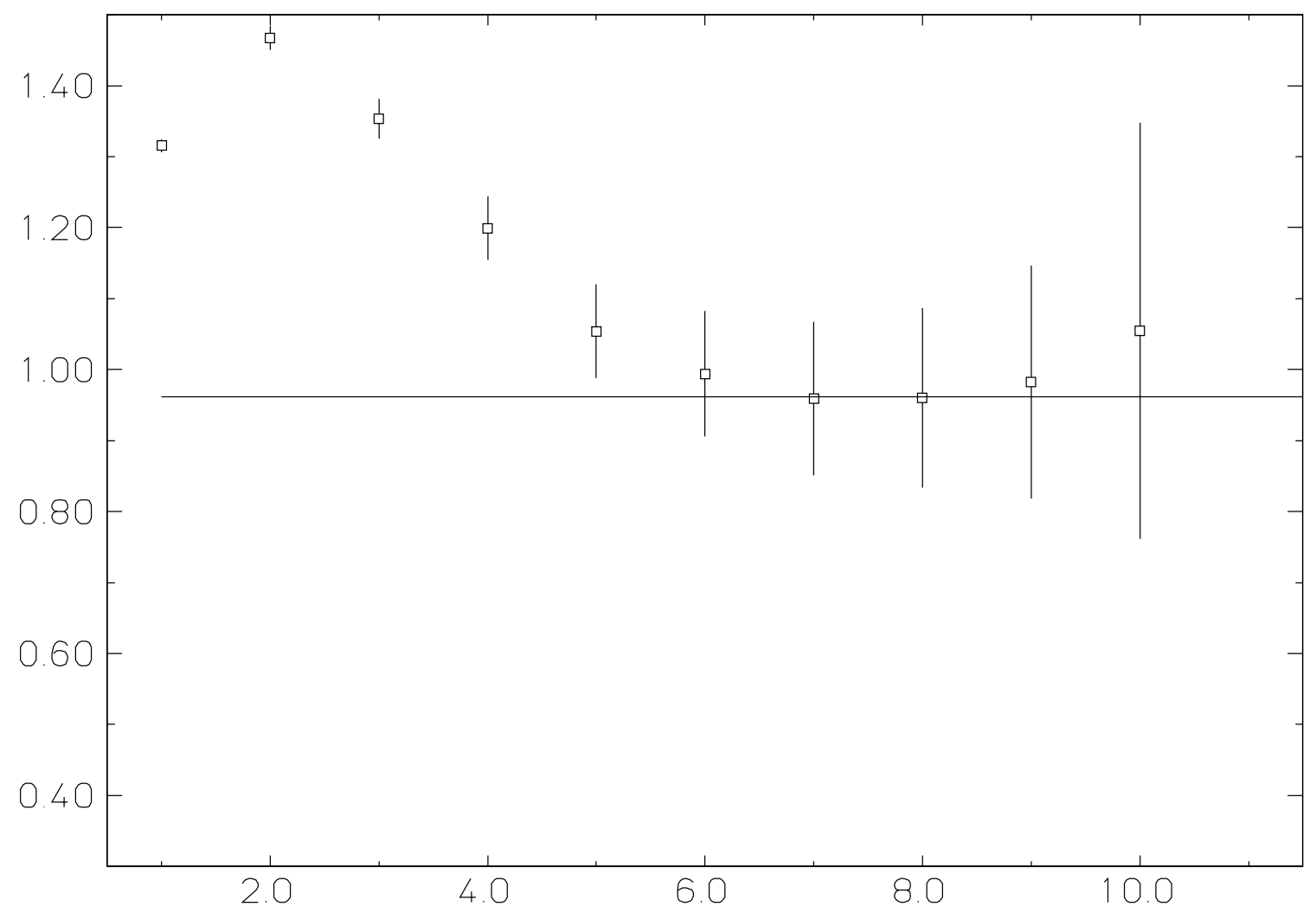

Fig. $4 \mathrm{~B}$ 


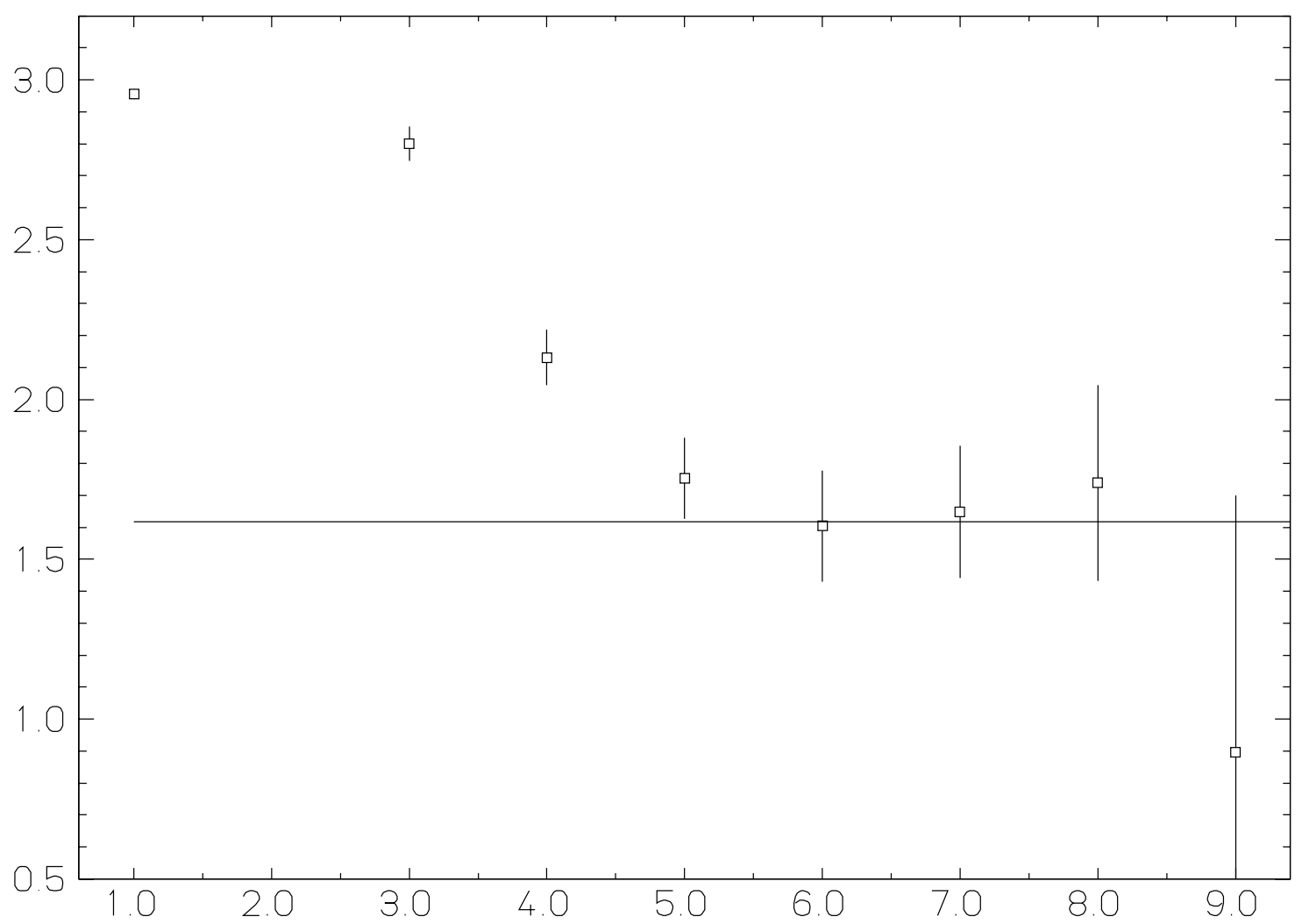

Fig. 4 C 


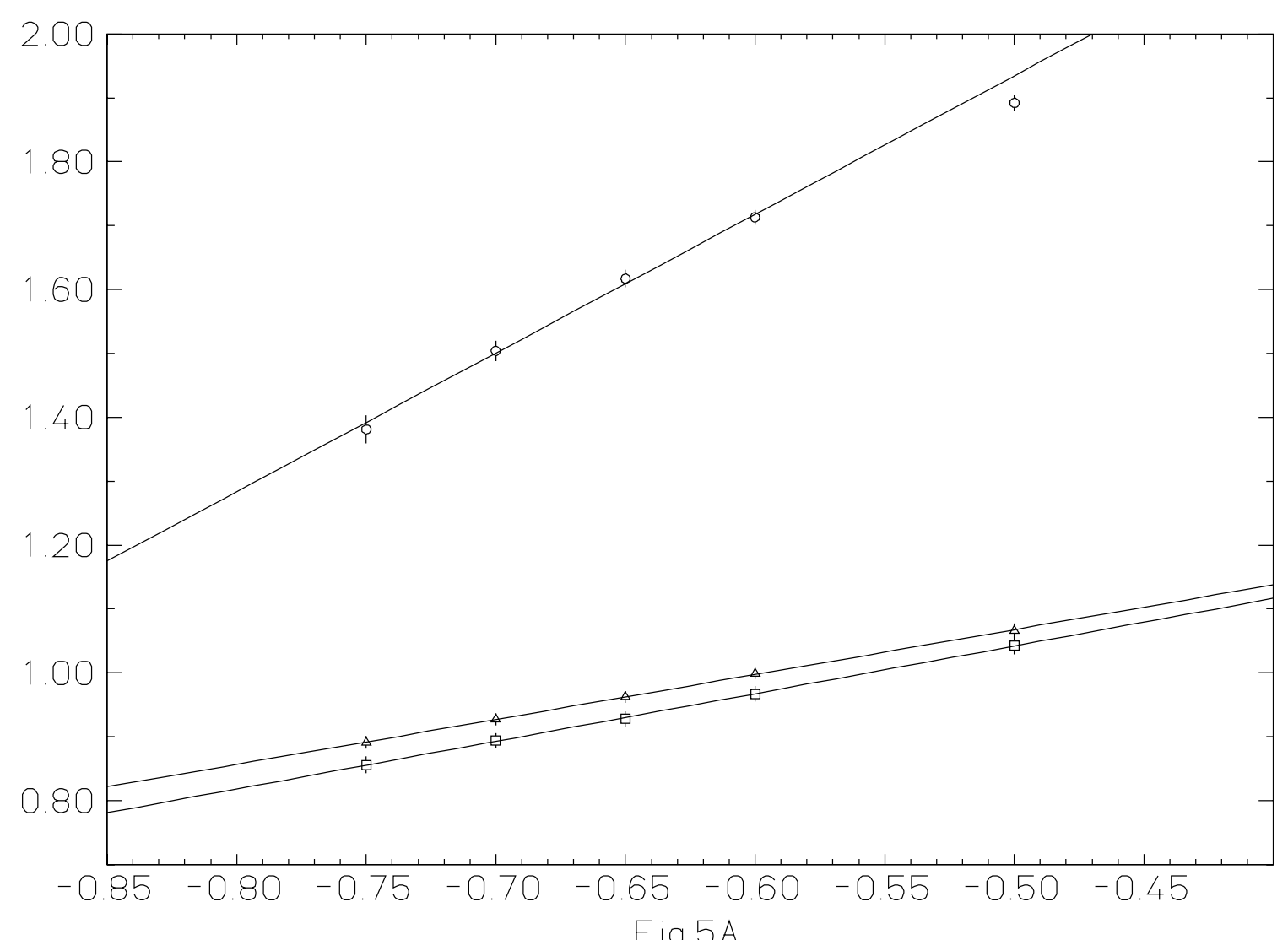

Fig. 5 A

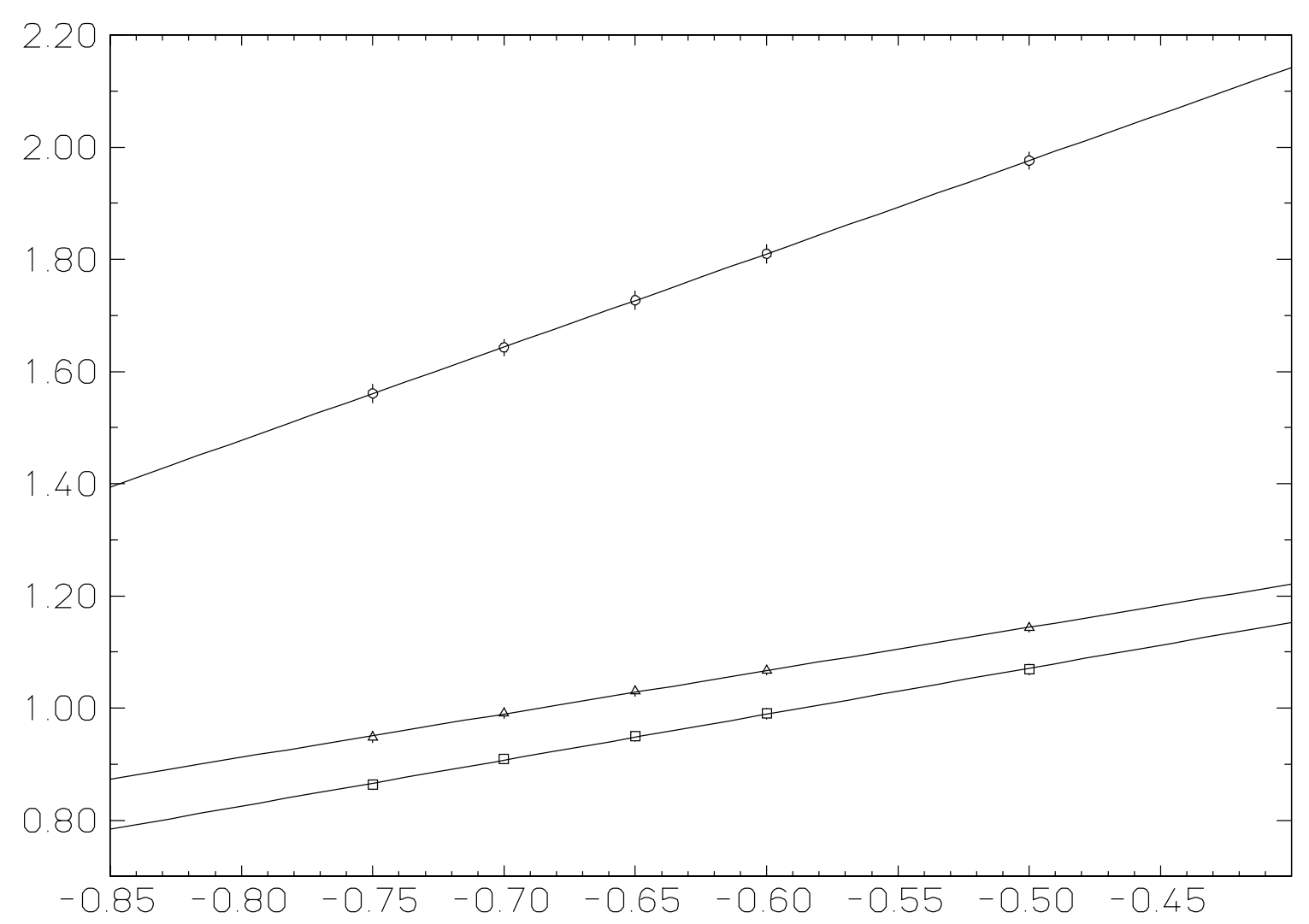

Fig. 5 B 